\title{
Misure di tenacità a frattura su acciai utilizzando velocità di deformazione elevate
}

\author{
Enrico Lucon \\ Institute of Nuclear Material Science, Centro di Studi Nucleari del Belgio, SCK•CEN, Boeretang 200, B-2400 \\ Mol (Belgio),e-mail: elucon@sckcen.be
}

RIASSUNTO. La conoscenza delle proprietà meccaniche di tipo dinamico per i materiali metallici è utile ogniqualvolta la sensibilità alla velocità di deformazione è di rilevanza per un acciaio, e qualora le condizioni reali di carico per una struttura (in caso di normale esercizio o di situazioni d'emergenza) siano diverse dal caso statico. Inoltre, in alcuni studi l'aumento della velocità di deformazione serve a simulare gli effetti di altri meccanismi di fragilizzazione quali l'invecchiamento termico (thermal ageing) o l'irraggiamento.

La presente memoria fornisce una panoramica dell'esperienza maturata al Centro Nucleare Belga $(\mathrm{SCK} \cdot \mathrm{CEN})$ nel campo delle misure di tenacità a frattura su acciai in condizioni di velocità di deformazione elevata, con particolare riguardo alle prove di resilienza strumentata su provini Charpy precriccati (PCVN).

Dopo una breve dissertazione sui meccanismi fondamentali che aiutano a comprendere gli effetti della velocità di deformazione sulla tenacità degli acciai in regime fragile e duttile, vengono presentate le procedure sperimentali ed analitiche per misurare la tenacità a frattura con velocità di deformazione elevata, prendendo in considerazione da un lato le principali normative internazionali (ASTM e ISO) e dall'altro il lavoro di normazione attualmente in corso sotto il coordinamento di SCK $\cdot \mathrm{CEN}$ : la revisione della norma ASTM E1921 (metodo della Master Curve per la misura della tenacità in regime di transizione duttile/fragile) e lo sviluppo di una nuova norma ISO sulle prove di tenacità dinamica con provini PCVN. Quest'ultimo documento è presentato in maggior dettaglio, concentrando l'attenzione sulla determinazione della tenacità dinamica in campo fragile con il metodo dell'Impact Response Curve e in campo duttile (curve di resistenza J-R) mediante l'uso di metodi mono- e multi-campione.

In conclusione, vengono presentati alcuni esempi tratti dalla banca dati sviluppata da SCK $\cdot \mathrm{CEN}$ per le misure di tenacità dinamica, prevalentemente su acciai da vessel di uso nucleare ( $R P V$ steels).

ABSTRACT. The knowlegde of dynamic mechanical properties is useful in all cases where the strain rate sensitivity of metallic materials is an issue, and whenever the actual loading conditions for a structure (either in normal operation or under accidental circumstances) are different from static. Furthermore, in some investigations increasing the loading rate is used to simulate other embrittling mechanisms such as thermal aging or neutron exposure.

This paper provides an overview of SCK $\cdot \mathrm{CEN}$ experience on measuring fracture toughness of steels at elevated loading rates, with specific emphasis on instrumented impact tests on precracked Charpy (PCVN) specimens.

After briefly dwelling on the basic mechanisms which explain loading rate effects on cleavage and ductile fracture toughness, the experimental and analytical procedures for measuring fracture toughness at elevated loading rates are addressed, both in terms of official ASTM and ISO test standards and considering standardization efforts currently in progress under SCK •CEN coordination: revision of ASTM E1921 (Master Curve methodology for measuring fracture toughness in the ductile-to-brittle transition region) and a future ISO standard on instrumented PCVN testing. This latter document is examined in more detail, focussing the attention on the dynamic evaluation of brittle fracture toughness (Impact Response Curve) and the determination of crack resistance curves using multiple and single-specimen techniques.

Finally, selected examples from SCK $\cdot \mathrm{CEN}$ database of dynamic toughness measurements will be illustrated, mainly relevant to reactor pressure vessel (RPV) steels.

\section{INTRODUZIONE}

In determinate circostanze, il tempo può diventare una variabile importante nelle problematiche relative allo studio della meccanica della frattura. In condizioni di ve- locità di deformazione ${ }^{1}$ elevata, due fenomeni possono influenzare e complicare la valutazione della tenacità a frattura dinamica per un materiale metallico:

\footnotetext{
${ }^{1}$ In questo lavoro, il termine "velocità di deformazione" è usato come sinonimo di "velocità di carico" o "velocità di caricamento" (traduzione letterale dell'inglese "loading rate").
} 
- la variazione delle proprietà meccaniche in funzione della velocità di deformazione (nella maggior parte dei metalli, le proprietà tensili aumentano sensibilmente quando la velocità di deformazione aumenta di alcuni ordini di grandezza);

- effetti inerziali, che possono assumere grande importanza quando la forza varia bruscamente o quando una cricca avanza rapidamente (parte del lavoro speso sul provino è convertita in energia cinetica).

La velocità di deformazione di un materiale metallico vicino all'apice di una cricca è di solito misurata come

$$
\dot{K}=\frac{d K_{l}}{d t}
$$

dove $K_{I}$ è il fattore di intensità delle sollecitazioni in modo $I$ e $t$ è la variabile tempo. Per velocità di deformazione fino a $K=10^{6} \mathrm{MPa} \sqrt{\mathrm{m}} / \mathrm{s}$, generalmente entrambi gli effetti sono significativi e devono essere presi in considerazione nelle misure di tenacità a frattura.

\section{EFFETTI QUALITATIVI DI UN AUMENTO DELLA VELOCITA’ DI DEFORMAZIONE}

Qualitativamente, le conseguenze di un aumento della velocità di deformazione sulla tenacità a frattura di un acciaio possono essere schematizzate nel modo seguente, in funzione del regime di frattura (fragile/transizione/duttile) nel quale il materiale si trova ad operare:

- comportamento fragile (lower shelf): un aumento della velocità di deformazione provoca una diminuzione della tenacità a frattura $\left(K_{I d}<K_{I c}\right)^{2}$;

- comportamento parzialmente fragile e parzialmente duttile (regime di transizione): la temperatura di transizione aumenta all'aumentare della velocità di deformazione $\left(T_{o, d}>T_{o, s}\right)^{3}$;

- comportamento duttile (upper shelf): una velocità di deformazione più elevata tende ad aumentare la resistenza del materiale all'innesco e alla propagazione duttile di una cricca $\left(J_{I d}>J_{I c}\right)$.

In termini grafici, le conseguenze di un aumento della velocità di deformazione sull'intera curva della tenacità a frattura in funzione della temperatura per un acciaio ferritico possono essere rappresentate come in Fig. 1.

In condizioni prevalentemente fragili, un aumento della velocità di deformazione rende più difficoltoso il movimento delle dislocazioni all'interno del materiale, con conseguente aumento dello sforzo di snervamento (indurimento, o hardening). Lo spostamento verso l'alto della curva di snervamento ha per effetto un aumento della temperatura alla quale si realizza la condizione di clivaggio, che corrisponde all'intersezione con la curva dello

\footnotetext{
${ }^{2}$ Il pedice "d" indica un parametro "dinamico".

${ }^{3}$ Il pedice "s" indica un parametro "statico".
}

sforzo di frattura (che dipende in maniera modesta dalla la velocità di deformazione o dalla temperatura). Tale incremento della temperatura di clivaggio corrisponde al fenomeno della fragilizzazione (embrittement), che può anche essere causato dall'irraggiamento neutronico. Quanto descritto è illustrato graficamente nella Fig. 2.

Anche quando il comportamento dell'acciaio è di tipo duttile, un incremento della velocità di deformazione rende più difficoltoso il movimento delle dislocazioni $\mathrm{e}$ di conseguenza la deformazione plastica del materiale. In tali circostanze, i fenomeni tipici associati all'innesco e alla propagazione duttile di una cricca (nucleazione, crescita e coalescenza di vuoti) avvengono a livelli più elevati di lavoro speso, e quindi la tenacità del materiale aumenta.

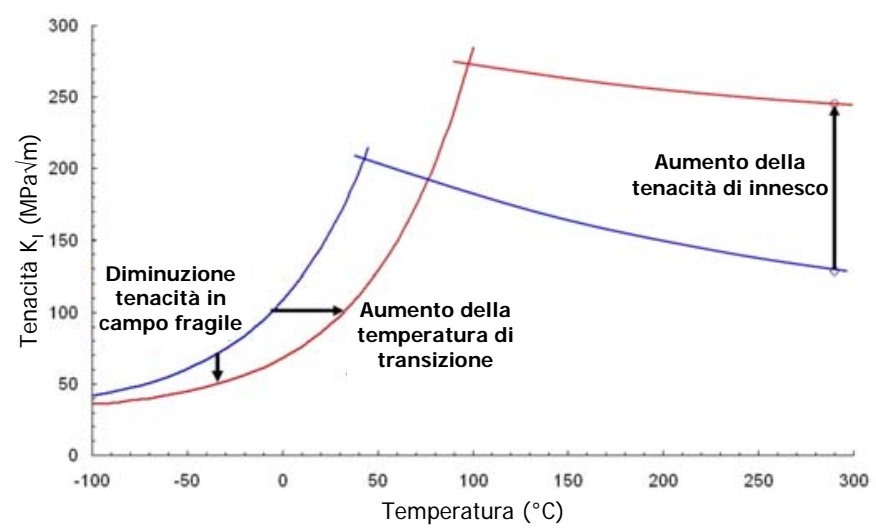

Figura 1. Effetto qualitativo di un aumento della velocità di deformazione sulla tenacità a frattura di un acciaio ferritico (curva blu: velocità quasi-statica; curva rossa: velocità dinamica).

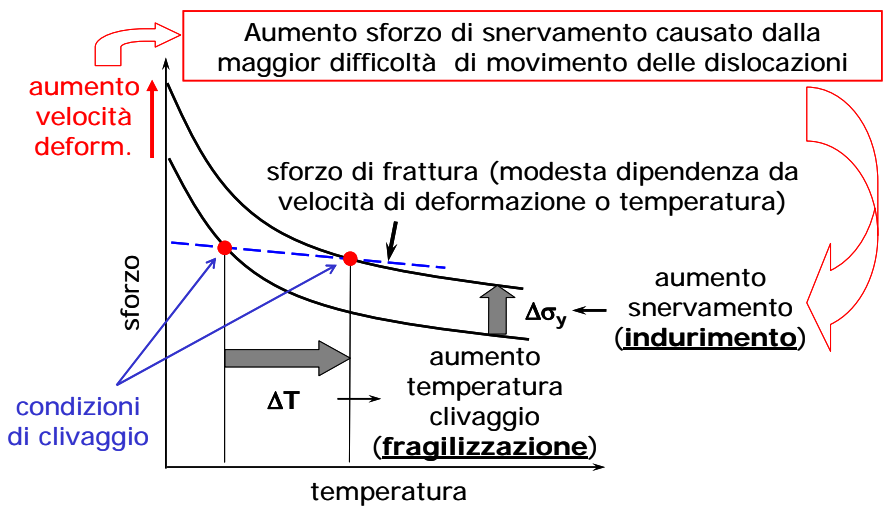

Figura 2. Effetti di indurimento e fragilizzazione causati da un aumento della velocità di deformazione in un acciaio con comportamento fragile.

\section{MISURA SPERIMENTALE DELLA TENACITA' A FRATTURA DINAMICA}

Nei paragrafi seguenti, si farà principalmente riferimento alle normative di prova già pubblicate $\mathrm{o}$ in via di pubblicazione da parte dell'ASTM (American Society for Testing and Materials) e dell'ISO (International Standardisation Organization). 


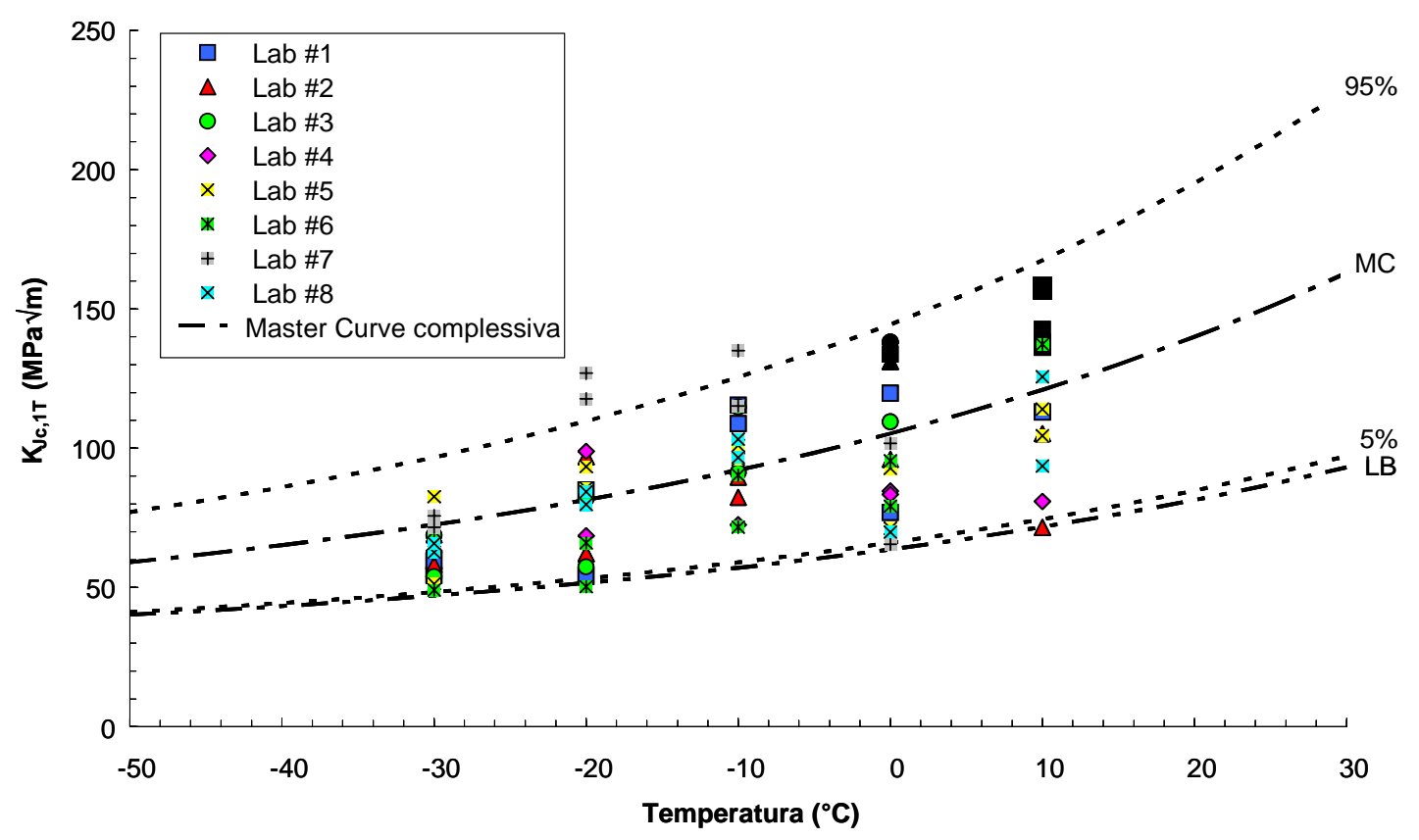

Figura 3. Prove di tenacità dinamica su provini Charpy precriccati dell'acciaio da vessel JRQ (A533B Cl.1), analizzati mediante il metodo della Master Curve. Dati del round-robin del CRP-8 dell'IAEA [12].

\subsection{Comportamento fragile (lower shelf)}

L'intervallo di velocità di deformazione per prove in condizioni quasi-statiche è definito come $0.55-2.75$ $\mathrm{MPa} \sqrt{\mathrm{m}} / \mathrm{s}$ nelle norme ASTM E399 [1] e E1820 [2] e come 0.55-3 MPa $\sqrt{\mathrm{m}} / \mathrm{s}$ nella norma ISO 12135 [3]. Il medesimo intervallo è prescritto anche dalla norma British Standard BS 7448:3 [4].

In caso di velocità di deformazione più elevate, le normative si limitano a raccomandare di determinare accuratamente i valori di forza applicata e prescrivono un tempo totale di prova non inferiore a $1 \mathrm{~ms}$. Inoltre, nei calcoli della tenacità a frattura si richiede l'uso di un valore di sforzo di snervamento corrispondente alla velocità di prova.

È interessante osservare che entrambe le norme ASTM (E399 e E1820) escludono la possibilità di eseguire prove di impatto utilizzando masse cadenti, quindi prove di resilienza Charpy o Pellini (drop weight). Inoltre, viene esplicitamente affermato che "diminuzioni significative della tenacità possono essere osservate all'aumentare della velocità di deformazione".

\subsection{Comportamento misto fragile/duttile}

La norma ASTM E1921 [5], che descrive la ben nota metodologia della Master Curve per la misura della tenacità a frattura di acciai ferritici in regime di transizione fragile/duttile, prevede che per una determinazione quasistatica della temperatura di riferimento $T_{o}$, la velocità di deformazione sia compresa tra 0.1 e $2 \mathrm{MPa} \sqrt{\mathrm{m}} / \mathrm{s}$. Tale requisito consente di limitare a un massimo di $10{ }^{\circ} \mathrm{C}$ la variazione di $T_{o}$ per effetto della velocità di prova [6]. È consentito altresì eseguire la prova al di sotto del limite inferiore $(0.1 \mathrm{MPa} \sqrt{\mathrm{m}} / \mathrm{s})$, purchè gli effetti ambientali siano trascurabili o assenti.
La versione attuale della norma (2005) non prevede la possibilità di eseguire prove con velocità di deformazione più elevate; tuttavia, l'autore della presente memoria sta preparando una revisione della E1921 nella quale sarà consentito applicare velocità di prova superiori, sino a poter testare provini Charpy precriccati utilizzando un pendolo strumentato [7-10].

Esiste infatti una consistente base sperimentale che dimostra senza ombra di dubbio che il metodo della Master Curve è pienamente applicabile alle misure di tenacità dinamica eseguite su provini Charpy precriccati; un chiaro esempio è fornito dalla Fig. 3, che mostra i risultati di un round-robin (prova interlaboratorio) recentemente organizzato nell'ambito del Coordinated Research Project Phase 8 (CRP-8) dell'IAEA (International Atomic Energy Agency) [11,12].

Nell'ambito del medesimo CRP-8, è attualmente in corso uno studio sulla sensibilità alla velocità di deformazione degli acciai ferritici [11]; lo studio si basa sulla raccolta ed analisi di valori della temperatura di riferimento $T_{o}$ ottenuti a diverse velocità di deformazione mediante la metodologia della Master Curve. La Fig. 4 mostra i risultati di prove eseguite da $\mathrm{SCK} \cdot \mathrm{CEN}$ su tre acciai (due acciai da vessel - JSPS e JRQ - e un acciaio ferritico/martensitico - E97) variando la velocità di prova; le pendenze variabili delle curve di regressione in scala semilogaritmica evidenziano la diversa sensibilità degli acciai considerati.

In assenza di misure sperimentali, una stima dell'incremento della temperatura di riferimento conseguente all'aumento della velocità di deformazione può essere ottenuta dalla seguente correlazione empirica, proposta da Wallin [13]: 
$T_{o, d}=\frac{T_{o, s} \cdot \Gamma}{\Gamma-\log (\dot{K})}$

dove la funzione $\Gamma$ è data da:

$\Gamma=9.9 \exp \left[\left(\frac{T_{o, s}}{190}\right)^{1.66}+\left(\frac{\sigma_{y s, T o s}}{722}\right)^{1.09}\right]$

con $\sigma_{y s, T o s}=$ limite di snervamento alla temperatura $T_{o, s}$ (temperatura di riferimento corrispondente a velocità di deformazione quasi-statiche). La correlazione (1) è stata sviluppata su 59 acciai, per velocità di prova comprese tra $10^{-1}$ e $10^{6} \mathrm{MPa} \sqrt{\mathrm{m}} / \mathrm{s}$, limiti di snervamento tra 200 e $1000 \mathrm{MPa}$ e valori $\mathrm{T}_{\mathrm{o}, \mathrm{s}}$ tra -180 e $0{ }^{\circ} \mathrm{C}$.

\subsection{Comportamento duttile (upper shelf)}

All'interno della norma ASTM E1820 [2], e più precisamente nell'Annex A14, vengono fornite indicazioni per l'esecuzione di prove con elevata velocità di deformazione.

Affinché la prova sia analizzabile, si richiede che la parte iniziale del grafico forza/spostamento sia sufficientemente ben definita e che la durata della prova non sia inferiore a un valore minimo $t_{w}$ che dipende dalla rigidità del provino e dalla massa totale del sistema di prova. Nel caso che la prova duri meno di $t_{w}$, le equazioni per il calcolo dell'integrale-J sono da considerarsi inaccurate.

Nel caso si debba determinare la curva di resistenza alla propagazione duttile ( $J-R$ curve), la norma raccomanda l'uso della tecnica della Normalizzazione (Normalization Data Reduction), descritta nell'Annex A15.

Da notare che nella norma E1820 si afferma esplicitamente che "la curva J-R e la tenacità critica $J_{I c}(t)$ aumentano al crescere della velocità di prova".

\section{LA FUTURA NORMA ISO SULLE PROVE DI TENACITA' DINAMICA UTILIZANDO PROVINI CHARPY PRECRICCATI}

In ambito ISO e con il coordinamento di $\mathrm{SCK} \cdot \mathrm{CEN}$, una normativa di prova è attualmente in preparazione per prove di tenacità dinamica su provini Charpy precriccati testati mediante un pendolo strumentato [14]. Tale norma è quasi interamente basata sull'ultima versione della procedura di prova scritta dal Comitato Tecnico TC5 dell'ESIS (European Structural Integrity Society) [15].

La norma tratta tutti e tre i regimi di comportamento a frattura (fragile / transizione / duttile), identificando il diagramma forza/tempo in base a quattro tipologie (Fig. 5):

- $\quad$ essenzialmente lineare elastico (tipo I);

- frattura fragile per clivaggio senza crescita duttile significativa ( $\Delta a \leq 0.2 \mathrm{~mm}$ ) (tipo II);

- frattura fragile per clivaggio con crescita duttile significativa $(0.2 \mathrm{~mm}<\Delta a \leq 1.6 \mathrm{~mm})$ (tipo III);

- frattura fragile per clivaggio con $\Delta a>1.6 \mathrm{~mm}$ o propagazione duttile in assenza di clivaggio (tipo IV).

\subsection{Provini}

Rispetto alle prove di tipo quasi-statico, la lunghezza minima ammessa della precriccatura di fatica è ridotta da

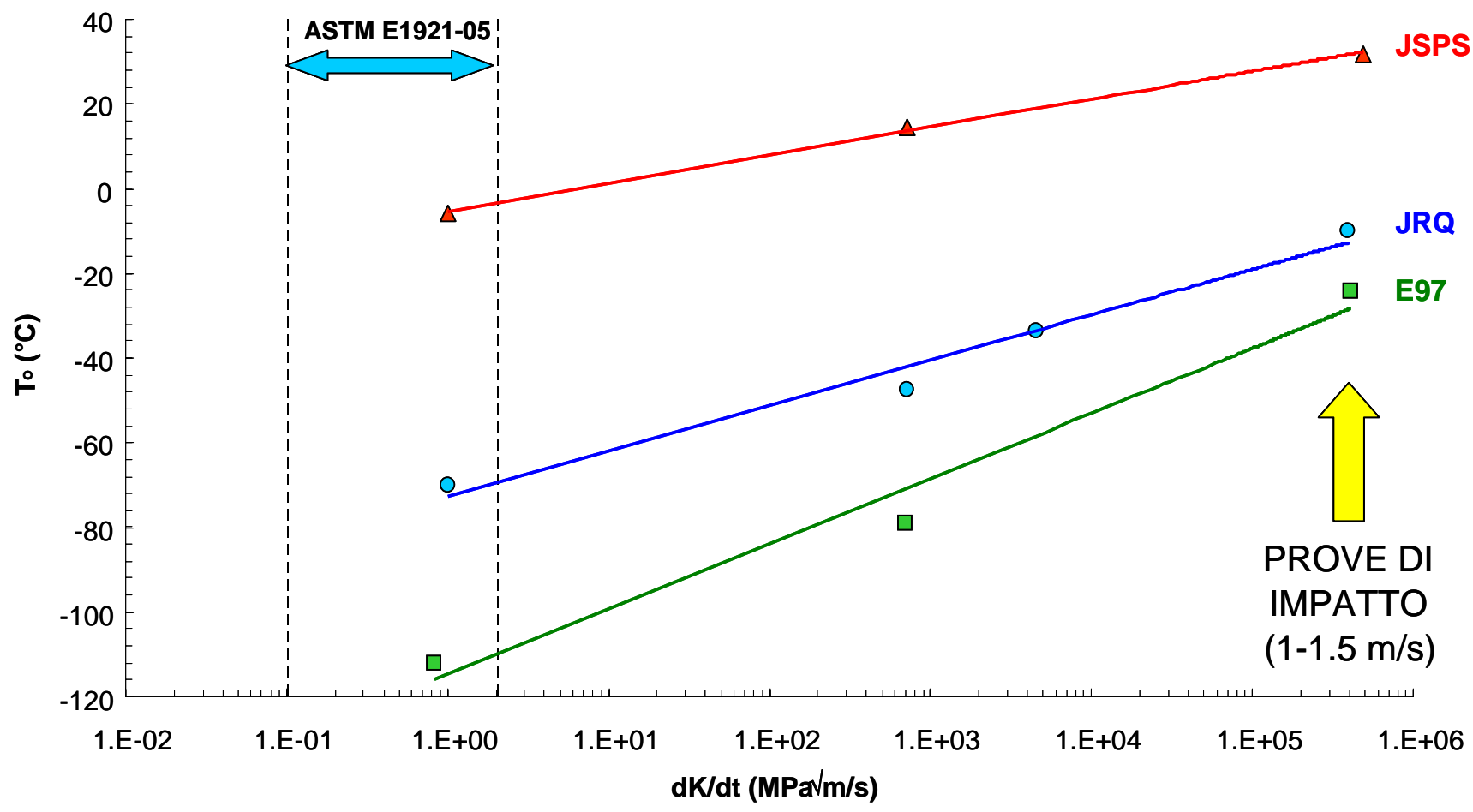

Figura 4 - Influenza della velocità di prova sulla temperatura di riferimento per due acciai da vessel (JSPS, JRQ) e un acciaio ferritico/martensitico (E97). Prove eseguite da SCK•CEN nell'ambito del CRP-8 dell'IAEA [11]. 

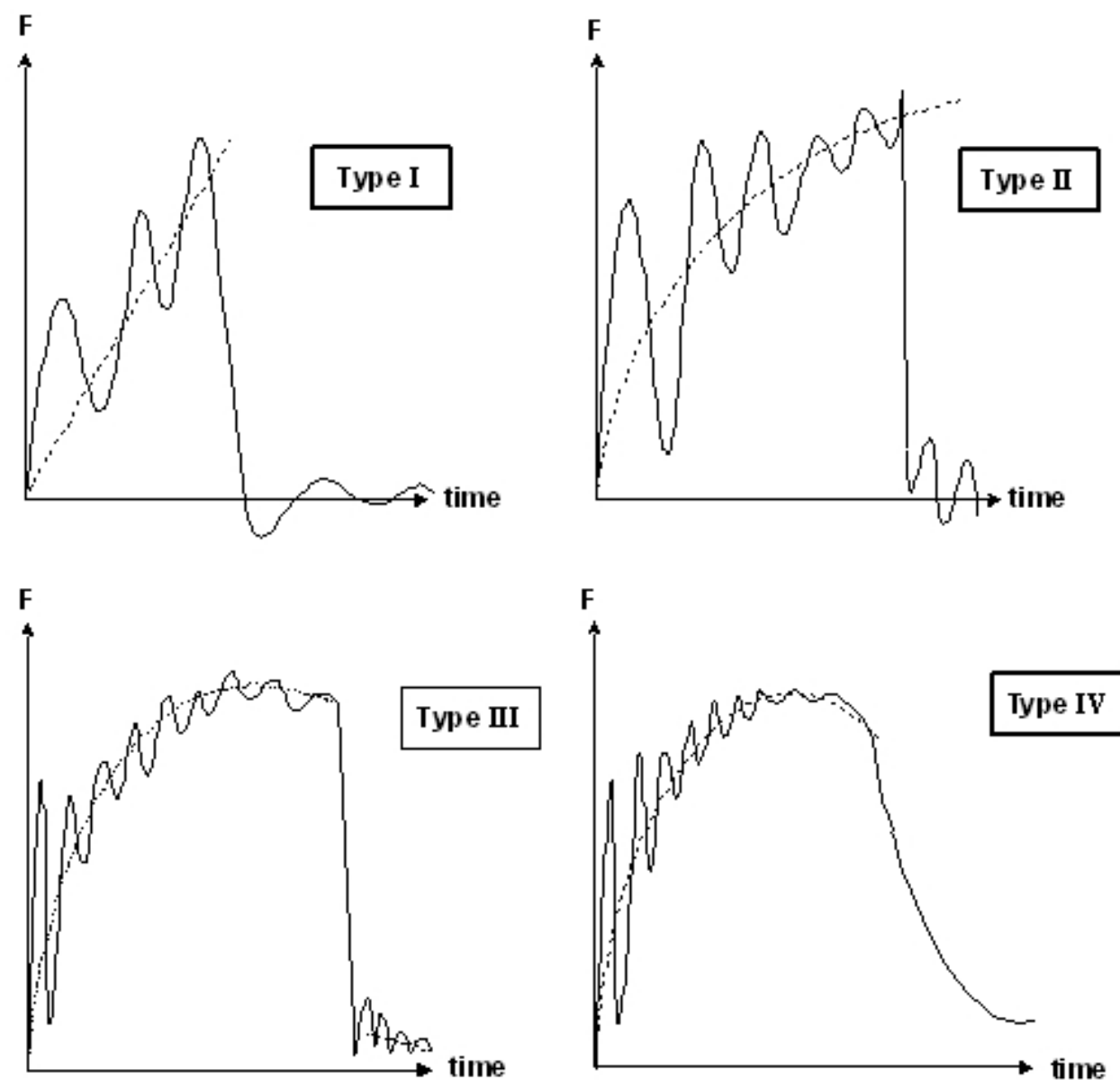

Figura 5. Possibili tipologie del diagramma forza/tempo per una prova di tenacità dinamica su provino Charpy precriccato.

$0.45 \mathrm{~W}$ a $0.3 \mathrm{~W}$, con $\mathrm{W}=$ larghezza del provino $(10 \mathrm{~mm})$. L'applicazione di scanalature laterali (side-grooves) è ammessa ed anzi raccomandata nel caso si voglia determinare la curva $J-R$.

\subsection{Macchine di prova}

Svariate tipologie di macchine di prova sono ammesse nella futura norma, benché l'attrezzatura consigliata sia un pendolo strumentato con angolo di caduta - e quindi velocità d'impatto - variabile. E' comunque consentito l'uso di macchine a massa cadente (drop-weight), macchine di prova di tipo servoidraulico, pendoli speciali con appoggi mobili e provino fisso ecc.

Il percussore (striker) strumentato può essere conforme alla geometria prevista sia dalla norma ISO 148-2 (raggio del coltello: $2 \mathrm{~mm}$ ) sia dalla norma ASTM E23 (raggio del coltello: $8 \mathrm{~mm}$ ).

\subsection{Velocità d'impatto}

Qualsiasi velocità d'impatto è consentita, fino a valori dell'ordine di $5 \mathrm{~m} / \mathrm{s}$ o superiori. Nel caso di un pendolo Charpy, la velocità di prova può essere ridotta sino a circa $1 \mathrm{~m} / \mathrm{s}$.

In caso di comportamento fragile, se la velocità è tale che il tempo di frattura è inferiore a 5 volte il periodo di oscillazione naturale del sistema provino/macchina $\left(t_{f}<\right.$ $5 \tau$ ), la tenacità a frattura non può essere calcolata a parti- re dal grafico forza/spostamento della prova; in tal caso, deve utilizzarsi il metodo dinamico di calcolo della tenacità dinamica dell'Impact Response Curve, descritto nella sezione successiva.

4.4 Metodo dinamico di calcolo della tenacità dinamica in regime di clivaggio

Il metodo detto Impact Response Curve (Curva di Risposta all'Impatto) [16], prevede la determinazione della tenacità dinamica $K_{I d}$ a partire dal tempo a frattura $t_{f}$, utilizzando una curva universale che è rappresentata dalla funzione

$K_{l d}=R v_{o} t^{\prime \prime}$

in cui: $R$ è funzione della cedevolezza della macchina di prova, $v_{o}$ è la velocità d'impatto e $t^{\prime \prime}$ è tabulato in funzione di $t_{f}$.

Per l'individuazione del tempo di frattura $t_{f}$, nell'ipotesi $t_{f}$ $<5 \tau$, si raccomanda di strumentare il provino con un estensimetro (strain gage) applicato in prossimità dell'apice della cricca (Fig. 6); una brusca diminuzione del segnale elettrico dell'estensimetro consente di individuare l'istante della frattura [17]. In alternativa, è possibile esaminare la variazione nel tempo di altri segnali, ad es. un segnale magnetico o la caduta di potenziale attraverso il provino percorso da corrente (Potential Drop). 


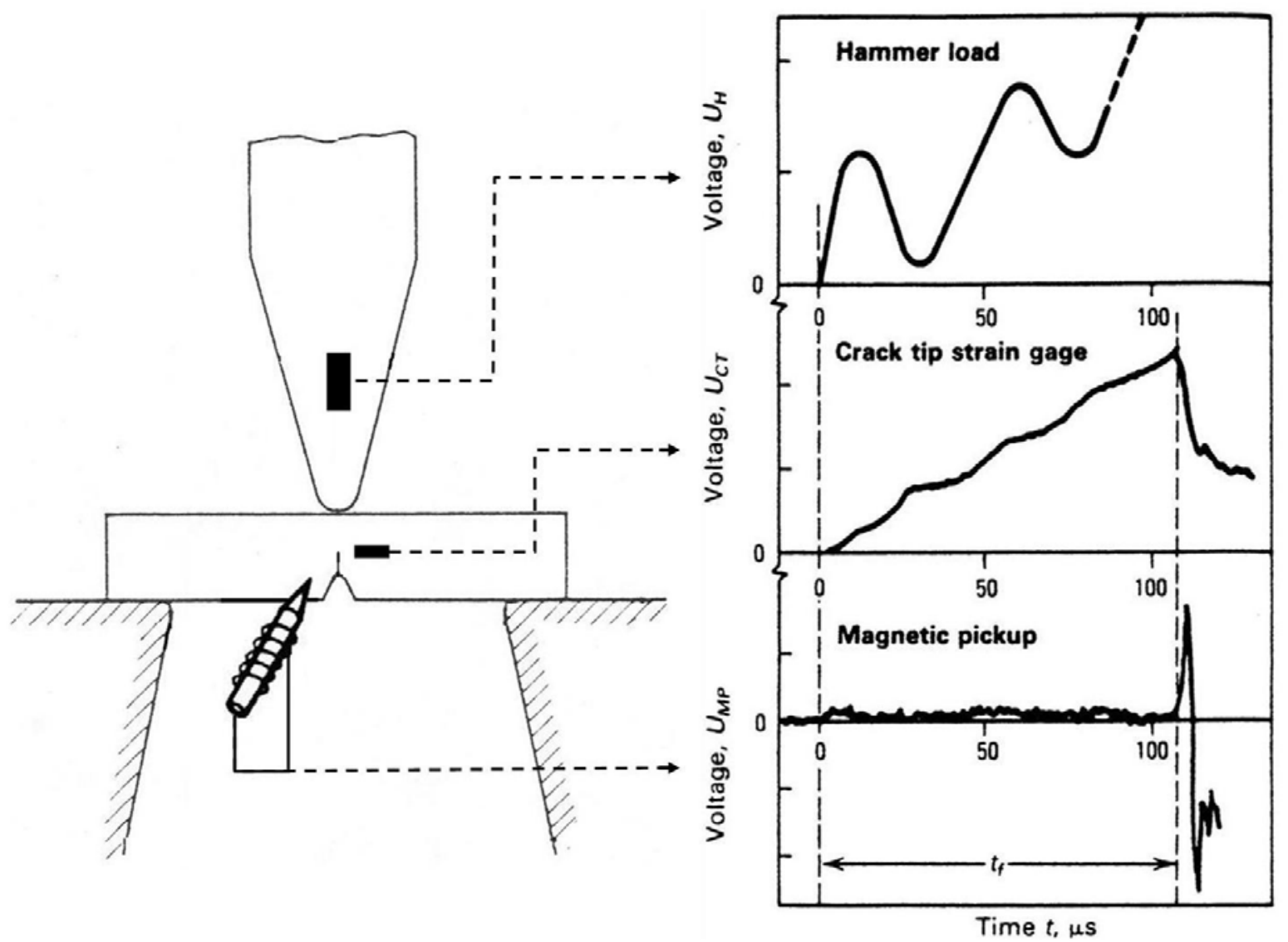

Figura 6. Esempi di metodi per l'individuazione del tempo di frattura in caso di frattura fragile.

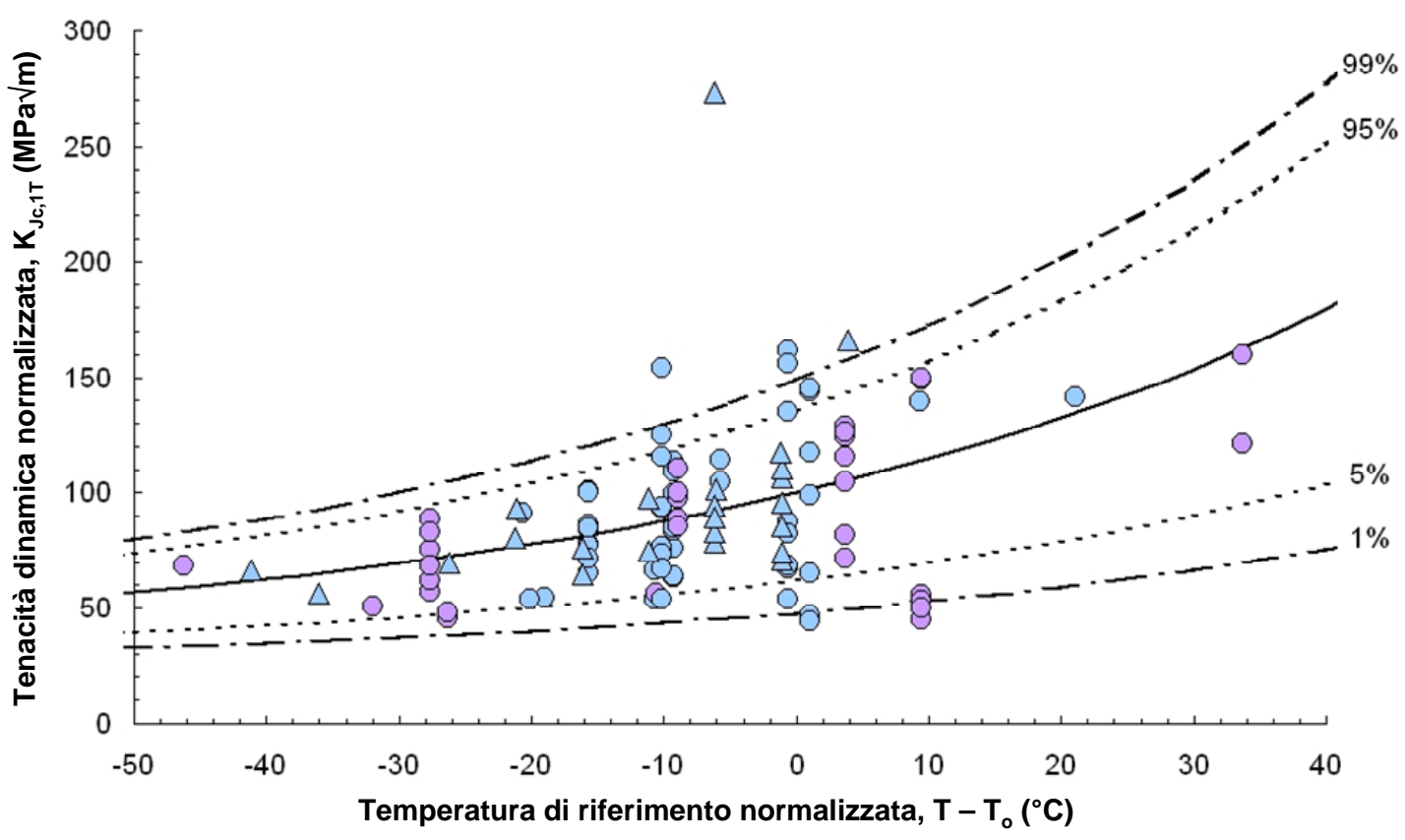

Figura 7. Banca dati SCK $\cdot \mathrm{CEN}$ costituita da misure di tenacità dinamica su provini PCVN di acciai da vessel (cerchi) e ferritici/martensitici (triangoli). Le prove, eseguite in regime di transizione fragile duttile, sono state analizzate mediante il metodo della Master Curve (rappresentata nella figura insieme a diversi livelli statistici di probabilità di frattura).

4.5 Metodi per la misura della tenacità dinamica in regime duttile

L'approccio consigliato per determinare la curva $J-R$ in condizioni dinamiche prevede l'uso di una metodologia di tipo multi-provino; la futura norma ISO cita i seguenti metodi di prova:

- Low-blow test: la velocità d'impatto viene variata da una prova all'altra in modo da ottenere quantità variabili di crescita duttile $\Delta a$; l'energia potenziale deve 

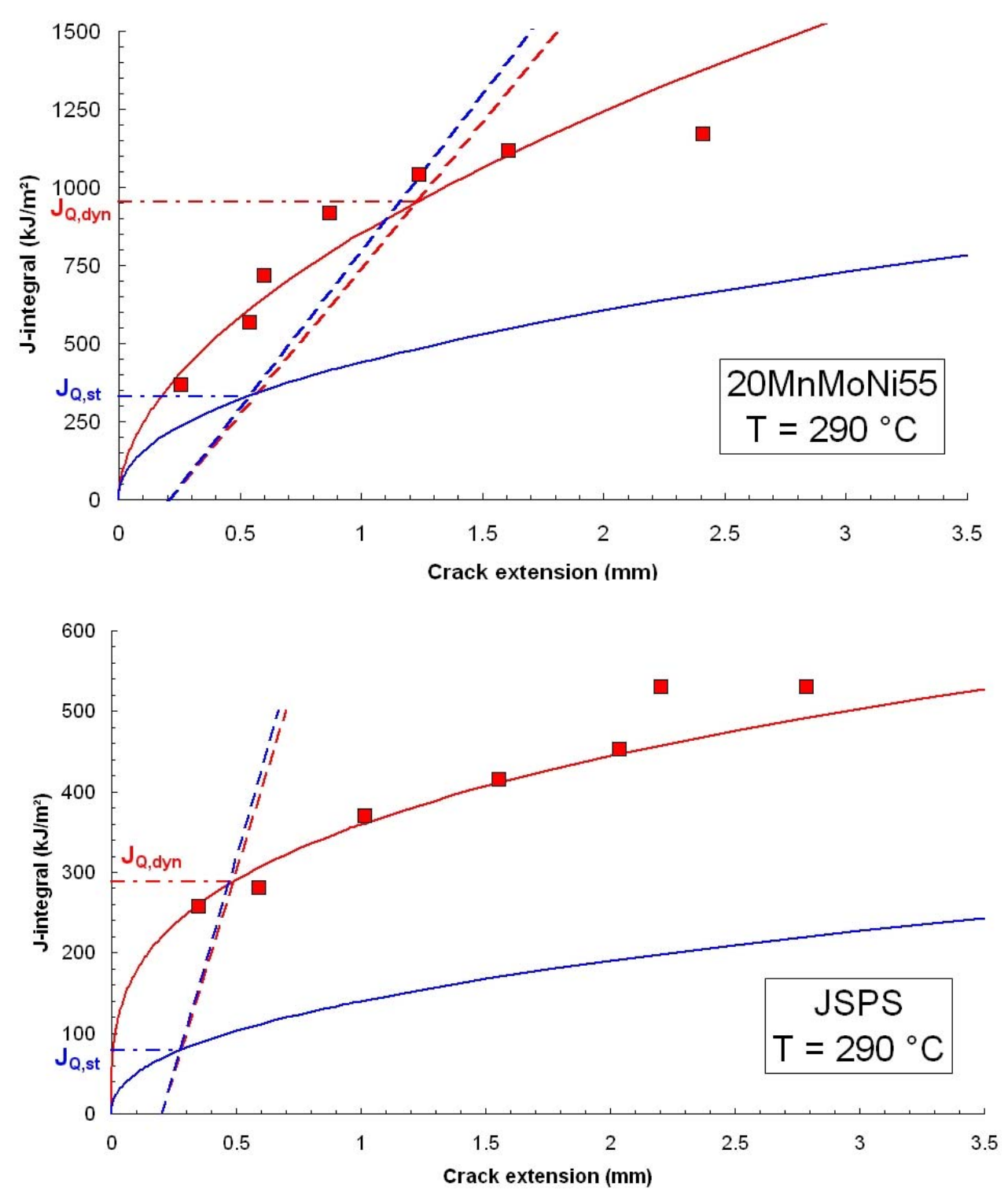

Figura 8. Misure di resistenza alla frattura duttile, ottenute per due acciai da vessel con velocità di deformazione quasi-statiche (curve blu) e dinamiche (curve rosse).

essere sufficiente a far propagare la cricca, senza tuttavia provocare la rottura completa del provino.

- Stop block test: valori differenti di crescita duttile tra un provino e l'altro vengono ottenuti variando la posizione d'arresto del martello, anche in questo caso evitando di rompere completamente il provino. Il metodo è tuttavia sconsigliato per macchine di prova standard, in quanto esiste il rischio di danneggiare la cella di carico.

- $\quad$ Cleavage R-curve method: le prove sono eseguite in regime di transizione fragile/duttile, facendo variare la temperatura di prova in modo da ottenere valori $\Delta a$ variabili. Le differenze tra le temperature di prova vengono ignorate [18].

In aggiunta, sono anche disponibili metodologie monoprovino, quali il metodo della Key Curve [19], l'approccio analitico a 3 parametri di Schindler [20] e la già citata tecnica della Normalizzazione descritta nella norma
ASTM E1820. Un recente studio eseguito da SCK •CEN ha evidenziato un buon accordo tra metodi multi- e mono-provino per alcuni tipici acciai da vessel [21].

\section{ESEMPI DI MISURE DI TENACITÀ DINAMICA TRATTI DALLA BANCA DATI $\mathrm{DI} \mathrm{SCK} \cdot \mathrm{CEN}$}

Per concludere, vengono presentati alcuni risultati di prove di tenacità dinamica eseguite da $\mathrm{SCK} \cdot \mathrm{CEN}$ su provini Charpy precriccati, provenienti in prevalenza da acciai da vessel per uso nucleare. La Fig. 7 mostra la nostra banca dati costituita da 113 prove condotte in regime di transizione fragile/duttile su 7 acciai in 11 diverse condizioni (per alcuni acciai, sono presenti misure su materiale sia non irraggiato che irraggiato). 
I valori di tenacità dinamica (normalizzati allo spessore di riferimento -1 pollice) sono rappresentati in funzione della differenza tra la temperatura di prova e la temperatura di riferimento $T_{o}$ calcolata per il relativo materiale; questo tipo di rappresentazione consente di riunire sullo stesso grafico materiali diversi tra loro. La Fig. 7 costituisce un'ulteriore dimostrazione che il metodo della Master Curve è perfettamente applicabile a misure di tenacità dinamica su provini PCVN.

Esempi di misure dinamiche di resistenza alla frattura duttile, ottenute mediante la metodologia Low Blow Test precedentemente descritta, sono mostrati per due tipici acciai da vessel nella Fig. 8, dove le curve dinamiche (in rosso) sono confrontate alle curve $J-R$ ottenute in condizioni quasi-statiche (in blu) alle stesse temperature di prova. La Fig. 8 consente di apprezzare chiaramente l'incremento della resistenza alla frattura duttile, conseguente ad un aumento della velocità di deformazione.

\section{BIBLIOGRAFIA}

[1] ASTM E399-05, Standard Test Method for LinearElastic Plane-Strain Fracture Toughness $K_{I c}$ of Metallic Materials, Annual Book of ASTM Standards 2006, Vol. 03.01 .

[2] ASTM E1820-05a, Standard Test Method for Measurement of Fracture Toughness, Annual Book of ASTM Standards 2006, Vol. 03.01.

[3] ISO 12135:2002, Metallic materials -- Unified method of test for the determination of quasistatic fracture toughness.

[4] BS 7448-3:2005, Method for determination of fracture toughness of metallic materials at rates of increase in stress intensity factor greater than $3.0 \mathrm{MPa} \sqrt{\mathrm{m}} / \mathrm{s}$, British Standards Institution.

[5] ASTM E1921-05, Standard Test Method for Determination of Reference Temperature, $T_{o}$, for Ferritic Steels in the Transition Range, Annual Book of ASTM Standards 2006, Vol. 03.01.

[6] J.B. Hall e K.K. Yoon, Quasi-Static Loading Rate Effect on the Master Curve Reference Temperature of Ferritic Steels and Implications, Proceedings of the 2003 ASME Pressure Vessels and Piping Conference (Cleveland, $\mathrm{OH}, 28-31$ luglio 2003).

[7] T.J. Koppenaal, Dynamic Fracture Toughness Measurements of High-Strength Steels Using Precracked Charpy Specimens, ASTM STP 563, 1974, pp. 92-117.

[8] K.R. Iyer and R.B. Miclot, Instrumented Charpy Testing for Determination of the J-Integral, ASTM STP 563, 1974, pp. 146-165.

[9] HTGR Fracture Toughness Program, EPRI NP-120, Project 337-1, Final Report, aprile 1976.

[10] Proceedings of C.S.N.I. Specialist Meeting on Instrumented Precracked Charpy Testing, EPRI NP-2102LD, Palo Alto, California, 1-3 dicembre 1980.

[11] H.-W. Viehrig e E. Lucon, IAEA Coordinated Research Project on Master Curve Approach to Monitor
Fracture Toughness of RPV Steels: Effect of Loading Rate, Proceedings of PVP2007, ASME Pressure Vessels and Piping Division Conference, 22-26 luglio 2007, San Antonio, Texas.

[12] E. Lucon e H.W. Viehrig, Round-Robin Exercise on Instrumented Impact Testing of Precracked Charpy Specimens (IAEA Coordinated Research Program Phase 8), Proceedings of PVP2007, ASME Pressure Vessels and Piping Division Conference, 22-26 luglio 2007, San Antonio, Texas.

[13] K. Wallin, Effect of Strain Rate on the Fracture Toughness Reference Temperature $T_{o}$ for Ferritic Steels, in: Recent Advances in Fracture (ed. R.K. Mahidhara et al.; The Minerals, Metals \& Materials Society, 1997).

[14] ISO TC 164/SC4 N465.3, Steel - Measurement of fracture toughness at impact loading rates using precracked Charpy V-notch test pieces, ultima revisione: 27 ottobre 2006.

[15] ESIS TC5, Proposed standard methods for instrumented pre-cracked Charpy impact testing of steels and other metallic materials, Draft 25.4: dicembre 2005.

[16] J.F. Kalthoff, S. Winkler and W. Böhme, A Novel Provedure for Measuring the Impact Fracture Toughness $K_{I d}$ with Precracked Charpy Specimens, Journal de Physique, Colloque $\mathrm{C} 5$, supplement au $\mathrm{n}^{\circ} 8$, Tome 46 , agosto 1985, pp 179-186.

[17] H. J. MacGillivray e D. F. Cannon, The Development of Standard Methods for Determining the Dynamic Fracture Toughness of Metallic Materials, ASTM STP 1130, 1992, pp. 161-179.

[18] W. Böhme, Experience with Instrumented Charpy Tests obtained by a DVM Round Robin and further Developments, ESIS 20, Ed. E. van Valle, MEP Publications, London, 1996, pp. 1-23.

[19] J.A. Joyce, Static and dynamic J-R curve testing of A533B steel using the Key-curve analysis technique, ASTM STP 791, 1983, pp. I-543-I560.

[20] H.J. Schindler, Estimation of fracture toughness from Charpy tests - theoretical relations, ASTM STP 1380, 1999, 340ff.

[21] E. Lucon, The Use of Single-Specimen Techniques for Measuring Upper Shelf Toughness Properties under Impact Loading Rates, rapporto BLG-1016, SCK $\cdot \mathrm{CEN}$, settembre 2005. 